\title{
Culture de cals de peupliers de la section Leuce et régénération de plantes entières
}

\author{
P Antonetti, J Pinon *
}

INRA, laboratoire de pathologie forestière, 54280 Seichamps, France

(Reçu le 23 août 1991; accepté le 27 juillet 1992)

\begin{abstract}
Résumé - La culture in vitro de cals et la régénération de plantes entières a été réussie chez une vingtaine de clones de peupliers représentant la plupart des espèces et hybrides de la section Leuce. Le milieu de Linsmaïer et Skoog modifié par Chalupa assure généralement la meilleure croissance des cals. La benzylaminopurine n'induit que rarement la néoformation de bourgeons et toujours à de faibles taux. À l'inverse, le thidiazuron assure la régénération de bourgeons chez tous les clones testés et seul un clone de Populus alba $\times$ P tremuloïdes requiert l'association de cette hormone avec la BAP. Les bourgeons néoformés s'enracinent aisément après repiquage sur le milieu de Murashige et Skoog modifié ou le Woody Plant Medium dépourvus d'hormone. La culture in vivo des plants obtenus ne pose aucun problème. Après un nombre important de subcultures des cals, on note une perte de l'aptitude organogénétique chez certains clones.
\end{abstract}

peuplier / Leuce / in vitro / cals / régénération

Summary - Callus culture of Leuce poplar and plant regeneration. Successful callus culture and regeneration of whole plants were achieved for 20 clones representing most of the poplar species and hybrids from the Leuce section (table 1). During the first subculture of calli from 8 clones, the frequency of necrosis was influenced by the nature of the media and its growth regulators. Generally, Linsmaier and Skoog media modified by Chalupa (and containing $10 \mu \mathrm{M} \mathrm{NAA}$ and $4.5 \mu \mathrm{M}$ $B A P)$ was suitable, but Wolter and Skoog media modified by Winton $(0.18 \mu \mathrm{M} 2,4-D$ and $4.5 \mu \mathrm{M}$ $B A P$ ) was more suitable for aspens (table II). The woody plant medium (WPM) was the least interesting medium for callus culture. Callus necrosis was more frequent on medium containing only BAP than on medium containing the hormones proposed by the above-mentioned authors. There was a positive correlation between thidiazuron concentrations (between $0-1 \mu \mathrm{M}$ ) and callus growth. When $B A P$ was mixed with thidiazuron, the positive effect of the latter hormone was significantly greater than the negative influence due to BAP (table V). Whatever the growth regulator used for the 18 clones, the following subcultures were free from necrosis (table III).

$B A P(2.5 \mu M)$ induced bud formation on callus of only 1 clone (Astria). However, thidiazuron promoted shoot formation on all clones except $\mathrm{P}$ alba $x \mathrm{P}$ tremuloides $c v$ 802-36-7. For most clones, optimal thidiazuron concentrations were 0.01 and $0.1 \mu \mathrm{M}$ (table IV). Regeneration of 802-36-7 was achieved by combining BAP and thidiazuron. Finally, a comparison conducted on 4 other clones showed that using thidiazuron alone showed better results in 2 of them while the 2 others produced more buds when BAP was associated with thidiazuron (table Vl).

After 2 yr $(\approx 20$ subcultures), 7 out of 14 clones had lost their ability to regenerate, at least using the hormone concentrations initially found to be the most suitable (table VII).

Neoformed shoots rooted easily when transferred onto hormone-free modified Murashige and Skoog medium (mainly P alba and its hybrids) or onto WPM (mainly for aspens). Regenerated plants were transferred and cultured in the nursery.

\section{poplar /Leuce / in vitro / callus / regeneration}

\footnotetext{
* Correspondance et tirés à part.
} 


\section{INTRODUCTION}

La maîtrise de la culture de cals et de la régénération de bourgeons constituent un préalable à toute étude de vitro-variation. Pour les différentes sections du genre Populus, de nombreux milieux de culture ont été proposés dans la littérature. Parmi ceux-ci, 3 ont été fréquemment utilisés : le milieu de Wolter et Skoog modifié par Winton pour $P$ tremuloides et $P$ tremula (Winton, 1968a, 1971), celui de Linsmaïer et Skoog modifié par Chalupa pour $P$ nigra, $P$ x euramericana, $P$ tremula et $P$ x canescens (Chalupa, 1974) et le Woody Plant Medium (WPM) (Lloyd, McCown, 1980) utilisé par Noh et al (1986) pour $P$ tremuloïdes et Russell et McCown (1986) pour $P$ alba $\times$ grandidentata et $P$ tremula.

Quelques travaux ont porté sur la néoformation de bourgeons sur les cals de peupliers de la section Leuce. Leur portée est cependant limitée par le nombre restreint de clones étudiés et le recours à la seule benzylaminopurine (BAP). Si celle-ci a permis l'obtention de nombreux bourgeons néoformés chez $P$ tremuloïdes (Noh et al, 1986) et $P$ alba (Kapusta et al, 1985), la plupart des auteurs n'ont obtenu que quelques bourgeons [Mathes, 1964; Wolter, 1968; Winton, $1968 \mathrm{~b}$ et 1970 (pour $P$ tremuloides) et Winton, 1971 (pour $P$ tremula)].

Récemment a été mis en évidence l'intérêt potentiel du thidiazuron [N-phényl-N'-(1, 2, 3-thiadiazol-5-yl] produit par Schering AG et doué d'une forte activité de type cytokinine (Mok et Mok, 1982). Nous conviendrons par la suite de l'abréviation TDZ pour cette substance. Elle a permis d'induire la néoformation de bourgeons sur des cals d'arbres fruitiers (Anonyme, 1986) et chez 2 clones de la section Leuce : $P$ alba $\times P$ grandidentata NC-5339 et $P$ tremula erecta (Russell et McCown, 1986 et 1988).

Nous avons donc cherché à maîtriser pour une large gamme de clones de la section Leuce, la culture de cals (en comparant différents milieux et hormones) et à induire la néoformation de bourgeons sur les cals en comparant l'intérêt respectif de la BAP et du thidiazuron.

\section{MATÉRIEL ET MÉTHODES}

\section{Clones}

La plupart des clones inclus dans cette étude sont issus du programme de sélection des peupliers de la section Leuce conduit à la Station d'amélioration des arbres forestiers de I'INRA (Lemoine, 1973). Nous avons complété cette gamme par Astria (tremble hybride triploïde sélectionné par Weisgerber en Allemagne) et NC5339 grisard (au sens large) d'origine nordaméricaine. Le tableau I précise la nature de chacun de ces clones. Tous ces ciones ont été cultivés en pépinière avant leur introduction in vitro.

\section{Méthodes}

\section{Conditions générales de culture}

La culture de cals in vitro a été initiée à partir d'entre-nœuds de rameaux aoûtés prélevés en pépinière. Après désinfection [(éthanol à $70 \%$ puis chlorure mercurique $(0,5 \%)]$ et rinçages à l'eau stérile, les rameaux ont été débarrassés de leur écorce pour mettre à nu le cambium puis découpés en tronçons. Ceux-ci ont été placés en boites de Petri sur les milieux de callogénèse (décrits ci-dessous). Les cultures ont été effectuées à $25^{\circ} \mathrm{C}$ sous une photopériode de $16 \mathrm{~h}$ (25 $\mu \mathrm{mol} \mathrm{m}{ }^{-2} \cdot \mathrm{s}^{-1}$ ) et après un mois le cambium avait suffisamment proliféré et pouvait être repiqué. Par cette technique, tous les clones testés (20 clones de la section Leuce) ont produit des cals. Les cultures ultérieures de cals ont été effectuées sous les mêmes conditions et étaient repiquées environ toutes les 6 semaines.

\section{Culture des cals}

Au premier repiquage (après obtention des cals à partir des rameaux), 8 clones ont été cultivés, 
Tableau I. Types botaniques des clones de la section Leuce dont des plantes entières ont été régénérées à partir de cals.

Type botanique

$P$ alba

P tremula

$P$ tremula $\times P$ tremuloides

$P$ tremuloides $\times P$ tremula

$P$ alba $\times P$ tremula

$P$ alba $\times P$ tremuloides

$P$ tremuloides $\times P$ alba

$P$ alba $\times P$ grandidentata
Clones

5972-S-38

FCO-F5

333-2, 333-4, 333-5, Astria

336-4

706-2, 706-8, 706-9, 706-10, 709-21, 709-27,

$710-21,710-23,712-1,717-1-\mathrm{B}-4$

802-36-7

808-111-6

NC-5339 à raison d'une centaine de cals par clone et par milieu, sur 3 milieux de callogénèse contenant les balances hormonales proposées par les auteurs :

- milieu de Wolter et Skoog modifié par Winton (1970) : 2,4-D $\left(0,18 \mu \mathrm{mol}^{-1} \mathrm{I}^{-1}\right)$ et BAP $(4,5$ $\left.\mu \mathrm{mol} . \mathrm{I}^{-1}\right)$.

- milieu de Linsmaïer et Skoog modifiè par Chalupa (1974) : ANA $\left(10 \mu \mathrm{mol} . \mathrm{I}^{-1}\right)$ et BAP $(4,5$ $\mu$ mol..$\left.^{-1}\right)$.

- WPM selon Russell et McCown (1986) avec ANA $\left(1 \mu \mathrm{mol}^{.1^{-1}}\right)$ et BAP $\left(0,45 \mu \mathrm{mol} . .^{-1}\right)$.

Après 6 semaines de culture, nous avons estimé le taux de nécrose : nombre de cals nécrosés / nombre total de cals mis en culture $\times 100$. Par cals nécrosés, nous entendons des cals n'ayant pas ou peu proliféré et dont la couleur est brun-noir indiquant qu'ils sont morts, alors que les cals vivants de peuplier sont jaunâtres à verdâtres.

Ultérieurement (au $9^{e}$ epiquage), pour tous les clones, le taux de nécrose et le développement pondéral moyen des cals sur le milieu de Linsmaïer et Skoog modifié par Chalupa ont été déterminés. Pour établir le gain de masse, les cals (30 à 100 selon les clones) étaient découpés manuellement en fragments d'environ $100 \mu \mathrm{g}$, puis mis en culture sur le milieu. Après 6 semaines de culture, leur masse était de nouveau déterminée par pesée et l'accroissement pondéral était déterminé par soustraction.

\section{Néoformation de bourgeons sur les cals}

Dans ce qui suit, le milieu utilisé correspond au milieu de base tel que défini par son auteur mais les balances hormonales sont celles que nous préciserons à chaque fois. Chaque essai est conduit au terme de 6 semaines. À ce stade, le taux de nécrose est estimé ainsi que le nombre moyen de bourgeons néoformés (nombre de bourgeons néoformés / nombre total de cals mis en culture).

Sur des jeunes lignées de cals (durée de culture des cals inférieure à 5 mois)

\section{Utilisation de la BAP}

Des essais préliminaires (Antonetti, 1987) conduits sur divers clones des sections Aigeiros et Tacamahaca avaient suggéré l'intérêt de la BAP, apportée à la concentration de $2,5 \mu \mathrm{mol}^{.} \mathrm{I}^{-1}$ après autoclavage des milieux, pour la néoformation de bourgeons (quelques clones de la section Leuce avaient été inclus dans cette expérience et seuls quelques cals de certains clones produisaient des bourgeons mais leur nombre était toujours très faible).

Nous avons donc étudié l'effet sur l'organogenèse (pour 8 clones) des 3 milieux précédents en remplaçant la balance hormonale d'origine par la BAP seule à 2,5 $\mu \mathrm{mol}^{.1^{-1}}$. Au premier repiquage, 70 cals ont été repiqués pour chaque couple clone $x$ milieu. 


\section{Utilisation du thidiazuron}

Vingt clones (à raison de 35 cals par clone et par concentration de thidiazuron) ont été cultivés sur le milieu de Linsmaïer et Skoog modifié par Chalupa additionné uniquement de thidiazuron. Celui-ci a été incorporé, après autoclavage, aux 3 concentrations de $0 ; 0,1$ et $1 \mu$ mol..$^{-1}$.

\section{Utilisation de l'association du thidiazuron et de la BAP}

Cinq clones (à raison de 28 cals par clone et par modalité) ont été cultivés sur le milieu de Linsmaïer et Skoog modifié par Chalupa en combinant cette fois les 2 hormones (thidiazuron et BAP) à différentes concentrations : 0 ; 0,$01 ; 0,1 ; 1$ et $10 \mu \mathrm{mol}^{-1} \mathrm{I}^{-1}$.

\section{Sur des lignées âgées de cals}

(durée de culture des cals de 2,5 ans)

Pour 14 clones, une soixantaine de cals appartenant à des lignées ayant subi une vingtaine de repiquages (soit environ 2,5 ans de culture) a été placée sur le milieu de Linsmaïer et Skoog modifié par Chalupa comportant les balances hormonales optimales pour la régénération de chacun de ces clones (balances hormonales définies pour des jeunes lignées de cals). Six semaines plus tard, le nombre de bourgeons néoformés a été noté.

\section{RÉSULTATS ET DISCUSSION}

\section{Culture des cals}

Le tableau II (colonnes de gauche) précise, pour 8 clones, le taux de nécrose des cals sur les 3 milieux de culture complets, au premier repiquage.

Tous clones confondus, ces 3 milieux ne se sont guère distingués après l'analyse statistique du fait d'une certaine interaction entre clones et milieux et entre types botaniques et milieux. Cependant certains traits communs ressortent de cette expérience même s'il aurait fallu, pour bien les mettre en évidence, intro- duire un nombre plus important de clones par hybrides et espèces. Ainsi pour l'ensemble des cals de trembles et de trembles hydrides, le taux de nécrose a été faible $(11,4 \%)$ sur le milieu de Wolter et Skoog modifié par Winton et plus élevé sur les 2 autres milieux $(44,5$ et $46,9 \%)$. À l'inverse, le peuplier blanc et ses hybrides ont subi peu de nécroses sur le milieu de Linsmaïer et Skoog modifié par Chalupa $(11,7 \%)$ alors que celles-ci ont été plus fréquentes sur les 2 autres milieux $(45,2$ et $46,2 \%$ ). De même, on note que pour chaque clone un milieu apparaît optimal : milieu de Wolter et Skoog modifié par Winton pour FCO-F5, 333-5, Astria (et aussi pour 333-4 bien que ce milieu n'entraîne pas la nécrose minimale pour ce clone), milieu de Linsmaïer et Skoog modifié par Chalupa pour 5972-S-38, 710-23, 333-4, 802-36-7 et WPM pour 706-8. Cependant, ces différences de taux de nécrose sont sûrement dues, outre la composition du milieu, aux différentes balances hormonales qui varient beaucoup d'un milieu à l'autre. Enfin, cette expérience a été réalisée au premier repiquage ce qui explique sûrement les forts taux de nécrose obtenus : en effet, au cours des repiquages successifs des cals, on note une acclimatation progressive des cals au milieu ce qui entraîne une diminution du taux de nécrose (que l'on peut remarquer dans le tableau III).

L'ajout de la BAP à la concentration de $2,5 \mu \mathrm{mol} . \mathrm{I}^{-1}$ dans les 3 milieux précédents (tableau II, colonnes de droite) a eu pour principal effet d'augmenter généralement le taux de nécrose par rapport à celui observé pour les mêmes clones sur ces milieux comportant leur balance hormonale habituelle (tableau II, colonnes de gauche). Ceci traduit une certaine toxicité de cette hormone bien que la BAP soit dans 2 cas sur 3 apportée en quantité moindre $\left(2,5 \mu \mathrm{mol}^{.} \mathrm{I}^{-1}\right.$ à la place de 4,5 $\left.\mu \mathrm{mol} . \mathrm{I}^{-1}\right)$ ce qui indiquerait un "effet protecteur' des auxines dans les milieux com- 
Tableau II. Influence de la nature des milieux de base et des hormones qui leur sont ajoutées sur le taux de nécrose des cals (en \%) des clones de la section Leuce lors du premier repiquage. En gras (colonnes de gauche) ou en souligné (colonnes de droite), les taux de nécrose les plus faibles pour chaque clone. Les valeurs suivies d'une même lettre en exposant ne diffèrent pas significativement au seuil de $5 \%$ au sein d'un clone : minuscules = test du 2 (Arbonnier, 1966), majuscules = test de Newmann et Keuls (après transformation des pourcentages par la relation $y=2 \operatorname{Arcsin} \sqrt{ } x$ ). Seul le clone Astria a produit des bourgeons : ${ }^{*}=0,04$ par cal et ${ }^{* \star}=0,03$.

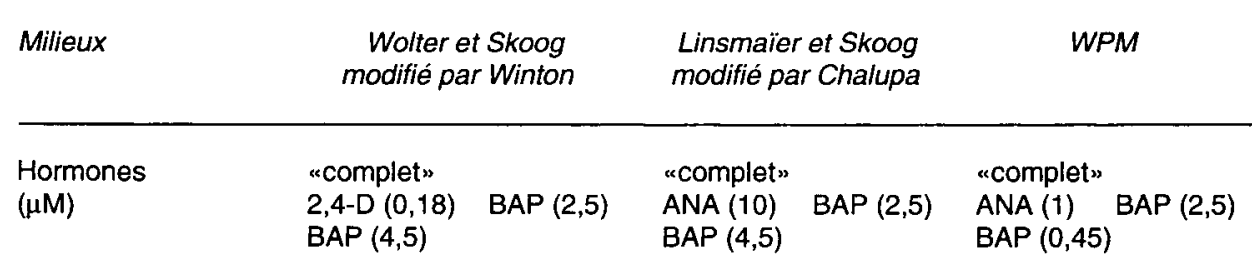

Clones

\begin{tabular}{|c|c|c|c|c|c|c|}
\hline FCO-F5 & $\mathrm{O}^{\mathrm{b}}$ & $67,1^{b^{\prime}}$ & $41,9^{a}$ & $78,6^{a^{a} b^{\prime}}$ & $49,5^{\mathrm{a}}$ & $85,7^{a^{\prime}}$ \\
\hline $333-4$ & $7,6^{b}$ & $80^{a}$ & $2,9 b$ & $34,3 c^{\prime}$ & 47,6 a & $61,4^{b}$ \\
\hline $333-5$ & $38,1^{b}$ & $21,4^{b^{\prime}}$ & $57,1^{a}$ & $61,4 a^{\prime}$ & $47,6 \mathrm{ab}$ & $28,6^{b^{\prime}}$ \\
\hline Astria & $0^{c}$ & $58,6^{b}$ * & $76,2^{a}$ & $85,7 a^{\prime}$ & $42,9^{b}$ & $40^{c} \star \star *$ \\
\hline Moyenne & $11,4^{\mathrm{A}}$ & $56,8 A^{\prime}$ & $44,5^{\mathrm{B}}$ & $65^{A^{\prime}}$ & $46,9^{B}$ & $53,9 \mathrm{~A}^{\prime}$ \\
\hline $5972-S 38$ & $51,4^{a}$ & $42,9 \mathrm{a}^{\prime}$ & 21,9 b & $15,7^{\circ}$ & $50,5^{a}$ & $21,4^{b^{\prime}}$ \\
\hline $706-8$ & 9,5 a & $17,1^{c}$ & $10,5^{a}$ & $32,9^{\circ}$ & $1,9 \mathrm{~b}$ & $52,9 \mathrm{a}^{\prime}$ \\
\hline $710-23$ & $59,1^{\text {a }}$ & $90^{a^{\prime}}$ & $0^{b}$ & $58,6^{c^{\prime}}$ & $57,1^{a}$ & $72,9 b^{\prime}$ \\
\hline $802-36-7$ & $61^{\mathrm{b}}$ & $70^{b^{\prime}}$ & $14,3^{c}$ & $51,4^{c^{\prime}}$ & $75,2^{a}$ & $88,6 \mathrm{a}^{\prime}$ \\
\hline Moyenne & $45,3^{A}$ & $55^{A^{\prime}}$ & $11,7^{\mathrm{A}}$ & $39,7^{A^{\prime}}$ & $46,2^{A}$ & $58,9^{A^{\prime}}$ \\
\hline Moyenne générale & $28,3^{A}$ & $55,9^{A^{\prime}}$ & $28,1^{A}$ & $52,3^{A^{\prime}}$ & $46,5^{A}$ & $56,5^{A^{\prime}}$ \\
\hline
\end{tabular}

plets. Nous avons remarqué également ce phénomène pour les peupliers des sections Aigeiros et Tacamahaca pour lesquels les cals supportent des quantités plus importantes de BAP si celle-ci est associée à une auxine (ANA ou 2,4-D) (Antonetti, 1990).

En conclusion, même s'il n'est pas toujours le milieu entraînant un taux de nécrose minimal, le milieu de Linsmaïer et Skoog modifié par Chalupa constitue un compromis acceptable pour la majorité des clones.

C'est pourquoi des cals appartenant à 20 clones ont été cultivés régulièrement sur ce milieu et lors du $9^{e}$ repiquage, nous avons estimé leur accroissement pondéral moyen ainsi que le taux de nécrose (tableau III). On remarque tout d'abord que les taux de nécrose ont beaucoup diminué par rapport au premier repiquage : il est nul pour 16 clones, très faible pour FCO- 
Tableau III. Accroissement pondéral moyen ( \pm erreur type) et taux de nécrose des cals de clones de peuplier de la section Leuce cultivés sur le milieu de Linsmaïer et Skoog modifié par Chalupa (après 6 semaines de culture au $9^{\mathrm{e}}$ repiquage).

\begin{tabular}{lcclrr}
\hline Clones & $\begin{array}{c}\text { Accroissement } \\
\text { pondéral } \\
\text { des cals }(\mathrm{mg})\end{array}$ & $\begin{array}{c}\text { Taux de } \\
\text { nécrose } \\
(\%)\end{array}$ & Clones & $\begin{array}{c}\text { Accroissement } \\
\text { pondéral } \\
\text { des cals }(\mathrm{mg})\end{array}$ & $\begin{array}{c}\text { Taux de } \\
\text { nécrose } \\
(\%)\end{array}$ \\
\hline $706-10$ & $637 \pm 147$ & 0 & $717-1-\mathrm{B} 4$ & $338 \pm 16$ & 0 \\
FCO-F5 & $630 \pm 331$ & 1,8 & $706-8$ & $305 \pm 13$ & 0 \\
$808-111-6$ & $613 \pm 55$ & 0 & $709-21$ & $235 \pm 148$ & 0 \\
$802-36-7$ & $570 \pm 32$ & 0 & $333-2$ & $190 \pm 51$ & 0 \\
$706-9$ & $555 \pm 255$ & 0 & $333-4$ & $182 \pm 9$ & 0 \\
$709-27$ & $544 \pm 33$ & 0 & $712-1$ & $165 \pm 15$ & 2,1 \\
$710-23$ & $479 \pm 57$ & 0 & $336-4$ & $160 \pm 30$ & 0 \\
NC-5339 & $362 \pm 67$ & 0 & $710-21$ & $151 \pm 17$ & 0 \\
$706-2$ & $358 \pm 72$ & 0 & Astria & $36 \pm 20$ & 18,7 \\
$5972-S 38$ & $349 \pm 15$ & 0 & $333-5$ & $23 \pm 5$ & 35,2 \\
\hline
\end{tabular}

F5 et 712-1 et modéré pour Astria et 3335. De plus, 18 de ces lignées ont doublé (ou plus) leur masse en 6 semaines, (seuls les cals d'Astria et de 333-5 n'ont guère proliféré), ce qui confirme l'intérêt de ce milieu pour la culture de cals.

\section{Néoformation de bourgeons sur les cals}

\section{Sur des jeunes lignées de cals}

\section{Utilisation de la BAP}

Sur les 8 clones cultivés en présence de BAP à 2,5 $\mu \mathrm{mol}^{-1} \mathrm{I}^{-1}$, seul Astria (tableau II) a produit des bourgeons (à un faible taux : 0,03 ou 0,04 bourgeon par cal selon le milieu). Les clones de la section Leuce présentent ainsi un comportement différent des clones des sections Aigeiros et Tacamahaca pour lesquels la BAP a toujours permis la néoformation de bourgeons sur les cals (Antonetti, 1990).

\section{Utilisation du thidiazuron}

Sur les 20 clones expérimentés, le thidiazuron a montré des effets avantageux pour 19 d'entre eux : diminution du taux de nécrose des cals et néoformation de bourgeons (tableau IV). Les nombres moyens de bourgeons néoformés varient beaucoup d'un clone à l'autre et sont compris entre 0,43 et 4,71 . Les nombres maximaux de bourgeons néoformés sont atteints pour la majorité des clones pour la concentration de $0,1 \mu \mathrm{mol}^{-1} \mathrm{I}^{-1}$ (tableau IV). La concentration optimale est de $0,01 \mu \mathrm{mol} . \mathrm{I}^{-1}$ pour FCO-F5, 5972-S38, 717-1-B4 et NC-5339. La concentration de $1 \mu \mathrm{mol}$. I $^{-1}$ est apparue plus avantageuse pour $336-4,710-21$ et $712-1$. Pour ces 2 derniers clones, seule cette concentration a permis la régénération et il se pourrait donc que celle-ci serait plus fréquente en élevant au delà de 1 $\mu \mathrm{mol} . \mathrm{I}^{-1}$ la concentration en thidiazuron. Seul le clone 802-36-7 n'a formé aucun bourgeon.

Enfin, on note pour tous les clones une forte prolifération des cals due à l'emploi 
Tableau IV. Influence de la concentration en thidiazuron sur la nécrose des cals de 20 clones et sur leur aptitude à former des bourgeons (milieu de Linsmaïer et Skoog modifié par Chalupa). Les valeurs suivies d'une même lettre ne sont pas significativement différentes au seuil de $5 \%$. * Valeurs moyennes établies sur les 20 clones.

\begin{tabular}{lcccc}
\hline & \multicolumn{4}{c}{ Concentration en thidiazuron $\left(\mu\right.$ mol..$\left.^{1}\right)$} \\
\cline { 2 - 5 } & 0 & 0,01 & 0,1 & 1 \\
\hline $\begin{array}{l}\text { Nombre de clones } \\
\text { avec nécroses }\end{array}$ & $16^{\mathrm{a}}$ & $6^{\mathrm{b}}$ & $9^{\mathrm{b}}$ & $6^{\mathrm{b}}$ \\
$\begin{array}{l}\text { Proportion moyenne } \\
\text { de nécroses (\%) }\end{array}$ & $18,8^{\mathrm{A}}$ & $2,8^{\mathrm{B}}$ & $3,7^{\mathrm{B}}$ & $2,7^{\mathrm{B}}$ \\
$\begin{array}{l}\text { Nombre de clones } \\
\text { avec des bourgeons }\end{array}$ & $0^{\mathrm{C}}$ & $11^{\mathrm{b}}$ & $17^{\mathrm{a}}$ & $13^{\mathrm{b}}$ \\
$\begin{array}{l}\text { Nombre moyen } \\
\text { de bourgeons }\end{array}$ & $0^{\mathrm{B}}$ & $1,24^{\mathrm{A}}$ & $1,61^{\mathrm{A}}$ & $0,85^{\mathrm{A}}$ \\
\hline
\end{tabular}

du thidiazuron (voir par exemple le tableau $\checkmark$ pour le clone 802-36-7).

\section{Utilisation mixte du thidiazuron et de la $B A P$}

L'apport de thidiazuron et de BAP à différentes concentrations a été testé sur le clone 802-36-7 pour lequel ces hormones prises séparément n'avaient pas conduit à la néoformation de bourgeons. Les taux de nécroses observés (tableau $V$ ) ont confirmé la faible toxicité du thidiazuron en dessous de $10 \mu \mathrm{mol} . \mathrm{I}^{-1}$ et, à l'inverse, celle plus prononcée de la BAP. Une seule balance $\left(0,1 \mu \mathrm{mol} .1^{-1}\right.$ de thidiazuron et 1 $\mu \mathrm{mol} . \mathrm{I}^{-1}$ de BAP) a permis d'obtenir quelques bourgeons chez le clone 802-36-7. Quatre autres clones, dont les cals produisaient des bourgeons en présence de thidiazuron seul, ont été soumis à l'association des 2 hormones. L'ajout de BAP s'est soldé généralement par une augmentation du nombre de bourgeons néoformés, ce qui nous conduit à proposer des balances hormonales ajustées à chacun de ces clones (tableau VI). On note que l'associa- tion de la BAP au thidiazuron est préférable pour optimiser la régénération de certains clones alors que pour d'autres, il vaut mieux utiliser le thidiazuron seul.

Il se confirme que l'accroissement pondéral des cals est fortement influencé par les régulateurs de croissance. Pour des concentrations en thidiazuron jusqu'à 1 $\mu \mathrm{mol} \mathrm{I}^{-1}$ et en BAP jusqu'à $10 \mu \mathrm{mol} . \mathrm{I}^{-1}$, le rôle de ces hormones se traduit par une valeur du $\mathrm{F}$ de Fisher significative à $1 \%$ et le coefficient de corrélation multiple s'élève à 0,73 . L'équation de régression a pour expression : $z=198+711 x-37 y, x$ étant la concentration en thidiazuron, $y$ celle en BAP et $z$ la masse des cals de 802-36-7. Les coefficients de régression traduisent un effet très positif du thidiazuron et un faible effet dépressif de la BAP.

\section{Sur des lignées âgées de cals}

Les cals de 7 clones (sur 14) cultivés depuis 2,5 ans (soit 20 repiquages) ont perdu leur aptitude à l'organogenèse (tableau VII), quel que soit leur type botanique. 
Tableau V. Influence de la teneur en thidiazuron et en BAP, ajoutés après autoclavage au milieu de Linsmaïer et Skoog modifié par Chalupa, sur le taux de nécrose (en \%) et l'accroissement pondéral (en $\mathrm{mg}$ ) des cals du clone 802-36-7. * Seul traitement ayant permis l'obtention de bourgeons $(0,39$ en moyenne).

$\begin{array}{llllll}\operatorname{TDZ} & 0 & 0,01 & 0,1 & 1 & 10 \\ \left(e n \mu m o l . .^{-1}\right) \\ B A P\end{array}$

$\begin{array}{llrrrrr}0 & \text { Nécrose } & 7,1 & 3,6 & 0 & 0 & 0 \\ & \text { Masse } & 37 & 233 & 721 & 1045 & 632 \\ 0,01 & & & & & \\ & \text { Nécrose } & 0 & 3,6 & 0 & 3,6 & 3,6 \\ & \text { Masse } & 23 & 137 & 160 & 832 & 339 \\ 0,1 & \text { Nécrose } & 7,1 & 10,7 & 0 & 0 & 0 \\ & \text { Masse } & 29 & 126 & 111 & 1102 & 422 \\ & & & & & & \\ 1 & \text { Nécrose } & 14,3 & 3,6 & 7,1 & 7,1 & 0 \\ & \text { Masse } & 91 & 140 & 293 * & 1097 & 266 \\ 10 & \text { Nécrose } & 100 & 92,9 & 89,3 & 100 & 100 \\ & \text { Masse } & 17 & 25 & 12 & 16 & 7\end{array}$

Cette perte du pouvoir organogène est soulignée par de nombreux auteurs et Bigot (1990) a résumé quelques uns de leurs travaux réalisés dans ce domaine.
Cependant, il est difficile de prouver si celle-ci est due à la dilution puis la perte lors des repiquages des secteurs organogènes du cal (les souches non organogènes ayant un taux de croissance supérieur), à des modifications de la constitution du cal au cours des cultures secondaires (disparition par exemple des descendances des assises compétentes, quand l'origine du cal est complexe), à des vitro-variations ou tout simplement à une modification des balances hormonales nécessaires à la néoformation de bourgeons.

\section{CONCLUSION}

La vingtaine de ciones incluse dans cette étude permet d'apprécier la variabilité des peupliers de la section Leuce pour la culture de cals et la néoformation de bourgeons.

Malgré des variations, le milieu de Linsmaïer et Skoog modifié par Chalupa additionné de ANA (10 $\left.\left.\mu \mathrm{mol}^{-1}\right)^{-1}\right)$ et BAP $(2,5$ $\mu$ mol. $^{-1}$ ) apparaît comme le milieu le plus polyvalent pour la callogénèse de l'ensemble des clones.

Les peupliers de la section Leuce présentent un caractère particulier pour la néoformation de bourgeons : la BAP n'a

Tableau VI. Nombre moyen de bourgeons néoformés sur les cals cultivés sur le milieu de Linsmaïer et Skoog modifié par Chalupa et en présence des hormones à leurs concentrations optimales. En gras : la balance hormonale la plus intéressante pour chaque clone.

\begin{tabular}{|c|c|c|c|c|}
\hline \multirow[b]{2}{*}{ Clones } & \multicolumn{4}{|c|}{$\begin{array}{l}\text { Nombre moyen de bourgeons obtenu } \\
\text { avec la meilleure concentration d"hormones }\left(\mu \text { mol. } .^{-1}\right)\end{array}$} \\
\hline & & $D Z$ seul & & $T D Z+B A P$ \\
\hline $\begin{array}{l}333-4 \\
5972-\mathrm{S} 38) \\
706-8 \\
717-1-\mathrm{B} 4 \\
802-36-7\end{array}$ & $\begin{array}{l}1,68 \\
1,73 \\
1,2 \\
3,97\end{array}$ & $\begin{array}{l}(T D Z=0,1) \\
(T D Z=0,01) \\
(T D Z=0,1) \\
(T D Z=\mathbf{0 , 0 1}) \\
0\end{array}$ & $\begin{array}{l}\mathbf{2 , 5 3} \\
1,29 \\
\mathbf{4 , 6 4} \\
3,39 \\
\mathbf{0 , 3 9}\end{array}$ & $\begin{array}{l}(\operatorname{TDZ}=\mathbf{0 , 1}+\mathrm{BAP}=\mathbf{0 , 0 1}) \\
(\mathrm{TDZ}=0,01+\mathrm{BAP}=0,1) \\
(\mathrm{TDZ}=\mathbf{0 , 1}+\mathrm{BAP}=\mathbf{0 , 0 1}) \\
(\mathrm{TDZ}=0,1+\mathrm{BAP}=0,1) \\
(\mathrm{TDZ}=\mathbf{0 , 1}+\mathrm{BAP}=\mathbf{1})\end{array}$ \\
\hline
\end{tabular}


Tableau VII. Nombre moyen de bourgeons néoformés par des cals cultivés depuis deux ans et demi sur le milieu de Linsmaïer et Skoog modifié par Chalupa. Les hormones (BAP et TDZ) sont aux concentrations optimales pour la néformation de bourgeons pour chaque clone.

Clones $\begin{gathered}\text { Nombre moyen de } \\ \text { bourgeons néoformés }\end{gathered}$

\begin{tabular}{ll}
\hline $709-27$ & 2,64 \\
FCO-F5 & 2,62 \\
$808-111-6$ & 1,78 \\
$706-8$ & 0,88 \\
$333-5$ & 0,78 \\
$709-21$ & 0,48 \\
Astria & 0,36 \\
$333-4,5972-S 38,710-23,712-1$ & 0 \\
$717-1-B 4,802-36-7, N C-5339$ & 0 \\
\hline
\end{tabular}

pratiquement pas d'effet alors que le thidiazuron est efficace, éventuellement associé à la BAP (ou obligatoirement pour les hybrides du type $P$ alba $\times P$ tremuloides). Le thidiazuron offre aussi l'avantage de stimuler la croissance des cals et d'être très peu toxique aux concentrations utilisées.

Enfin, l'enracinement (sur milieu de Murashige et Skoog modifié sans hormone ou WPM sans hormone) et l'acclimatation des pousses néoformées ne posent pas de problèmes (Antonetti, 1990).

\section{REMERCIEMENTS}

Les auteurs remercient $M$ Lemoine, $M$ Terrasson et $J$ Russell pour la fourniture du matériel végétal ainsi que $B$ Liche (Schering) pour celle du thidiazuron.

\section{RÉFÉRENCES}

Anonyme (1986) Dropp (Thidiazuron) cotton defoliant. Technical information, 4th edition, Schering agrochemicals, Berlin, $19 p$
Antonetti $P$ (1987) Recherche in vitro de peupliers résistants aux toxines d'Hypoxylon mammatum. DEA Sci Agron, INPL-ENSAIA, Nancy, $40 p$

Antonetti $P$ (1990) Contribution à l'étude de la culture in vitro et de la vitro-variation du genre Populus. Thèse Univ Nancy $1 n^{\circ} 2470$, $337 \mathrm{p}$

Arbonnier $P$ (1966) L'analyse de l'information. Aperçu théorique et application à la loi multinomiale. Ann Sci For 23, 949-1017

Bigot C (1990) Apport de la culture in vitro dans le contrôle de la néoformation. In: Cinquantenaire de la culture in vitro, Versailles (France), 24-25 oct 1989, les Coll INRA $n^{\circ}$ 51, INRA Paris, 37-53

Chalupa V (1974) Control of root and shoot formation and production of trees from Poplar callus. Biol Plant 16, 316-320

Kapusta J, Skibinska A (1985) Introduction of morphogenesis and regeneration in the callus of Populus alba $\mathrm{L}$ and $P$ nigra $\mathrm{L}$. $J$ Tree Sci 4, 34-38

Lemoine $M$ (1973) Amélioration des peupliers de la section Leuce sur sols hydromorphes. Thèse de docteur ingénieur, Univ Nancy $I n^{\circ}$ $8205,120 \mathrm{p}$

Lloyd G, Mc Cown B (1980) Commerically feasible micropropagation of Mountain laurel, Kalmia latifolia, by use of shoot tip culture. Int Plant propagators' Soc Combined Proc For $30,421-427$

Mathes M (1964) The in vitro formation of plantlets from isolated aspen tissue. Phyton 21, 137-141

Mok MC, Mok DWS (1982) Cytokinin activity of N-phenyl-N'-1, 2, 3-thidiazol-5-yl urea (thidiazuron). Phytochemistry 21, 1506-1511

Noh EW, Sussah C (1986) High efficiency shoot regeneration from callus of quaking aspen. Plant Cell Rep 5, 464-467

Russell JA, Mc Cown BH (1986) Culture and regeneration of Populus leaf protoplasts isolated from non seedling tissue. Plant Sci 46 , 133-142

Russell JA, Mc Cown BH (1988) Recovery of plants from leaf protoplasts of hybrid poplar and aspen clones. Plant Cell Rep 7, 59-62

Winton LL (1968a) The initiation of fiable aspen callus. Phyton 25, 15-21 
Winton LL (1968b) Plantlets from aspen tissue cultures. Science 160, 1234-1235

Winton LL (1970) Shoot and tree production from aspen tissue cultures. Am J Bot 57, 904-909
Winton LL (1971) Tissue culture propagation of European Aspen. For Sci 17, 348-350

Wolter KE (1968) Root and shoot initiation in aspen callus cultures. Nature 219, 509-510 